expression and mutation signatures may provide the means of ultimately matching patients with treatment and matching treatment with response mechanisms. Given that relapsed disease appears to be chemoresistant across multiple classes of therapy, integration of personalized treatment is likely to be most effective when applied as early as possible.

\section{References}

1. Cancer Genome Atlas Research Network. Genomic and epigenomic landscapes of adult de novo acute myeloid leukemia. N Engl J Med. 2013;368(22):2059-2074.

2. Papaemmanuil E, Gerstung M, Bullinger L, et al. Genomic classification and prognosis in acute myeloid leukemia. N Engl J Med. 2016;374(23):2209-2221.

3. Welch JS, Ley TJ, Link DC, et al. The origin and evolution of mutations in acute myeloid leukemia. Cell. 2012;150(2):264-278.

4. Klco JM, Spencer DH, Miller CA, et al. Functional heterogeneity of genetically defined subclones in acute myeloid leukemia. Cancer Cell. 2014;25(3):379-392.

5. Krevvata M, Shan X, Zhou C, et al. Cytokines increase engraftment of human acute myeloid leukemia cells in immunocompromised mice but not engraftment of human myelodysplastic syndrome cells. Haematologica. 2018;103(6):959-971.

6. Tyner JW, Tognon CE, Bottomly D, et al. Functional genomic landscape of acute myeloid leukaemia. Nature. 2018;562(7728):526-531.

7. Klco JM, Spencer DH, Lamprecht TL, et al. Genomic impact of transient low-dose decitabine treatment on primary AML cells. Blood. 2013;121(9):1633-1643.

8. Kurtz SE, Eide CA, Kaempf A, et al. Molecularly targeted drug combi- nations demonstrate selective effectiveness for myeloid- and lymphoid-derived hematologic malignancies. Proc Natl Acad Sci U S A. 2017;114(36):E7554-E7563.

9. Malani D, Murumagi A, Yadav B, et al. Enhanced sensitivity to glucocorticoids in cytarabine-resistant AML. Leukemia. 2017;31(5):11871195.

10. Tavor S, Shalit T, Ilani NC, et al. Dasatinib response in acute myeloid leukemia is correlated with FLT3/ITD, PTPN11 mutations and a unique gene expression signature. Haematologica. 2020;105(12):27952804.

11. Estey EH. Treatment of relapsed and refractory acute myelogenous leukemia. Leukemia. 2000;14(3):476-479.

12. DiNardo CD, Rausch CR, Bent on C, et al. Clinical experience with the BCL2-inhibitor venetoclax in combination therapy for relapsed and refractory acute myeloid leukemia and related myeloid malignancies. Am J Hematol. 2018;93(3):401-407.

13. Marcucci G, Geyer S, Laumann K, et al. Combination of dasatinib with chemotherapy in previously untreated core binding factor acute myeloid leukemia: CALGB 10801. Blood Adv. 2020;4(4):696-705.

14. Ravandi F, O'Brien S, Thomas D, et al. First report of phase 2 study of dasatinib with hyper-CVAD for the frontline treatment of patients with Philadelphia chromosome-positive $(\mathrm{Ph}+)$ acute lymphoblastic leukemia. Blood. 2010;116(12):2070-2077

15. Paschka P, Schlenk RF, Weber D, et al. Adding dasatinib to intensive treatment in core-binding factor acute myeloid leukemia-results of the AMLSG 11-08 trial. Leukemia. 2018;32(7):1621-1630.

16. Ayatollahi H, Shajiei A, Sadeghian MH, et al. Prognostic importance of C-KIT mutations in core binding factor acute myeloid leukemia: a systematic review. Hematol Oncol Stem Cell Ther. 2017;10(1):1-7.

17. Sekeres MA, Elson P, Kalaycio ME, et al. Time from diagnosis to treatment initiation predicts survival in younger, but not older, acute myeloid leukemia patients. Blood. 2009;113(1):28-36.

18. Stone RM, Mandrekar SJ, Sanford BL, et al. Midostaurin plus Chemotherapy for acute myeloid leukemia with a FLT3 mutation. N Engl J Med. 2017;377(5):454-464.

\title{
Convalescent plasma for administration of passive antibodies against viral agents
}

\section{Giovanni Di Minno, ${ }^{1}$ Pier Mannuccio Mannucci, ${ }^{2}$ James W. Ironside, ${ }^{3}$ Carlo Federico Perno, ${ }^{4}$ Lutz Gürtler ${ }^{5}$ and Louis Aledort ${ }^{6}$}

${ }^{1}$ Dipartimento di Medicina Clinica e Chirurgia, Centro Hub per le Emocoagulopatie, Napoli, Italy; ${ }^{2}$ Fondazione IRCCS Ca' Granda Ospedale Maggiore Policlinico, Angelo Bianchi Bonomi Hemophilia and Thrombosis Center, Milan, Italy; ${ }^{3}$ Centre for Clinical Brain Sciences, University of Edinburgh, Edinburgh, UK; ${ }^{4}$ Departmental Medicine Laboratory, ASST Niguarda Hospital, Milan, Italy; ${ }^{5}$ Max von Pettenkofer Institute, Ludwig Maximilians University of Munich, Munich, Germany and 'Mary Weinfeld Professor of Clinical Research in Hemophilia at the Icahn School of Medicine at Mount Sinai, New York City, NY, USA

E-mail: GIOVANNI DI MINNO - diminno@unina.it

doi:10.3324/haematol.2020.267427

A dministration of passive antibodies through transfusion of plasma from donors recovering from a viral infection has long been employed to treat individuals infected with the same pathogen. ${ }^{1}$ However, in studies with convalescent plasma (CP), differences and inherent limitations (e.g., sensitivity/specificity of tests to quantify neutralizing antibodies; sample size; scheduling of treatment [early/late CP administration vs. degree of disease severity], the presence of confounders [concomitant treatments]), and restricted generalizability of data argued for large-scale, randomized, controlled trials. ${ }^{1,2}$ The results of a multicenter proof-of-concept, observational Italian study in 46 patients with moderate or severe acute respiratory distress syndrome due to infection with the novel coronavirus, SAR-CoV-2, who needed mechanical ventilation and/or continuous positive airway pressure are reported in this issue of the Journal. ${ }^{3}$ The interval between symptom onset and study inclusion was highly variable (2-29 days). The 7 day mortality rate was $6 \%$ in patients given CP compared with an expected $15 \%$ according to Italian statistics and $30 \%$ in a small concurrent cohort not treated with CP. Weaning from continuous positive airway pressure was achieved in 26 of 30 patients, and three of the seven intubated patients were extubated. Whether those who received $\mathrm{CP}$ earlier improved more or faster than patients who received plasma later in the course of the disease is not clarified, nor are the reasons for administering one, two or three CP bags provided. In this larger than previous uncontrolled reports, five serious adverse events (including 1 transfusion-related acute lung injury [TRALI]) occurred in four patients. Although TRALI may be triggered by transfused antibodies, ${ }^{4}$ CP was safe in this study as it was in 
Table 1. Summary on the use of convalescent plasma\# in moderately to severely ill patients infected with major emerging viruses.

Virus, Ref(s) Design/Patients/Treatments/Objectives Results/Interpretation/Limitations

\section{SARS-CoV-2}

JAMA. 2020;323(16):1582-1589.
Case series. 5 critically ill patients (36-65 years old, 2 women), all with confirmed SARS-CoV-2 infection + ARDS, rapidly progressing severe pneumonia and high viral load despite treatment; all receiving mechanical ventilation; $\mathrm{PaO}_{2} / \mathrm{FiO}_{2}<300$. 10-22 days after admission (mean, 18.2), patients received $400 \mathrm{~mL}$ CP with a SARS-CoV-2-specific IgG binding titer $\geq 1: 1000$ and a neutralization titer $\geq 40$.

SARS-CoV-2

Proc Natl Acad Sci U S A. 2020; 117(17):9490-9496.

Chest. 2020;158(1):e9-e13.

SARS-CoV-2

JAMA 2020;324(5):460-470.

\section{SARS-CoV-2}

Haematologica

2020;105(12):2834-2840.

Multicenter, one-arm, interventional study. 46 patients, mean 62 years old (SD 11), 28 males (61\%), confirmed SARS-CoV-2 infection + moderate-to-severe ARDS, elevated CRP and need
Prospective observational. 10 severely ill patients (34-78 years old) with confirmed COVID-19 infection. Maximal supportive care $+200 \mathrm{~mL}$ CP (neutralizing antibody titers $>1: 640$ ) given to the patients 16.5 days (median) after onset of illness. Primary endpoint: CP safety. Secondary endpoints: improvement of symptoms and laboratory parameters within 3 days after administration of CP.

Case reports. 4 critically ill patients (31-73 years old) with confirmed SARS-CoV-2 infection and respiratory failure requiring mechanical ventilation (ECMO in 2 cases) were given 200-2400 mL of CP 11-18 days (mean: 15.25 days) after admission.

Open-label, multicenter, randomized trial. The original sample size was 100 for each group to provide $80 \%$ power with a two-sided significance level of $\alpha=0.05$.

Patients (males/females, $\geq 18$ years of age) with confirmed COVID-19 and severe ARDS and/or hypoxemia or life-threatening conditions (shock, organ failure, or requiring mechanical ventilation) were stratified by age and disease severity. Intervention: 4-13 mL/kg volume of ABO-compatible CP with IgG titer $\geq 1: 640$ + standard treatment ( $v$ s. standard treatment alone). Primary outcome: time to improvement within 28 days, secondary outcomes: 28-day mortality, time to discharge, negativity of viral PCR within $72 \mathrm{~h}$ of treatment. The study was terminated early due to the containment of the SARS-CoV-2 epidemic in China.
Following CP transfusion, body temperature normalized within 3 days (4/5 patients). Within 12 days, the SOFA score decreased; $\mathrm{PaO}_{2} / \mathrm{FiO}_{2}$ increased (range, 284-366 after vs. 172-276 before); viral loads became negative, and SARS-CoV-2-specific and neutralizing antibody titers increased (range, 1:80-1:320 on day 7 vs. 1:40-1:60 before). ARDS resolved in 4 patients 12 days after transfusion, 3 patients stopped mechanical ventilation within 2 weeks of treatment. $3 / 5$ patients were discharged from the hospital, 2 in a stable condition 37 days after CP.

By day 3 after CP transfusion, improved clinical symptoms and laboratory values, increases in neutralizing antibody titers, patients' oxygen saturation and lymphocyte count; decreases in CRP, SARS-Cov-2 viral load, and radiological lung lesions (varying degrees of absorption of lung lesions within 7 days). No severe adverse events following CP administration.

All recovered from the infection. Resolution or partial absorption of lung lesions (all cases); reduced viral load (2 cases), 3/4 discharged between days 18-43.

Recovery/discharge within 1 to 4 weeks after starting CP transfusion; one patient discharged on supplemental oxygen, another required continued critical care for multi-organ failure. At the time of the termination, $103 / 200$ patients (58.3\% males, median age 70 years) had been enrolled. Of these, $98.1 \%$ (101/103) completed the trial. Within 28 days, clinical improvement was detected in $51.9 \%$ (27/52) in the CP group vs. $43.1 \%(22 / 51)$ in the control group (HR, 1.40 [95\% Cl: $0.79-2.49] ; P=0.26)$. Among those with severe disease, improvement was found in $91.3 \%$ (21/23) of the CP group $v$ s. $68.2 \%(15 / 22)$ in the control group (HR, 2.15 [95\% CI: $1.07-4.32] ; P=0.03)$. Among those with life-threatening disease, improvement was found in $20.7 \%$ (6/29) of the CP group $v$. $24.1 \%$ (7/29) in the control group (HR, 0.88 [95\% CI: 0.30-2.63]; $P=0.83)$. At day 28 , there was no difference in mortality $(15.7 \%$ vs. 24.0\%; OR, 0.65 [95\% CI: $0.29-1.46] ; P=0.30)$ or time to discharge (51.0\% vs. 36.0\%; HR, 1.61 [95\% CI: 0.88-2.93]; $P=0.12$ ). $\mathrm{CP}$ treatment was associated with a higher conversion rate to negative viral PCR at $72 \mathrm{~h}(87.2 \%$ in the $\mathrm{CP}$ group vs. $37.5 \%$ in the control group (OR, 11.39 [95\% CI: 3.91 33.18]; $P<0.001)$. Two adverse events occurred in two patients in the CP group.

Patients had been symptomatic for a mean of 14 days for mechanical ventilation and/or CPAP. 1-3 units (250-300 mL each) of CP (neutralizing antibody titers: 1:80-1:320) +usual treatment. Primary outcome: 7-day hospital mortality. Secondary outcomes: $\mathrm{PaO}_{2} / \mathrm{FiO}_{2}$, laboratory/radiologic changes, weaning from mechanical ventilation, $\mathrm{CP}$ safety.

Randomized, double-blind phase III prospective trial. Patients of all ages with severe influenza A infection; onset of illness within 6 days of randomization. Randomization based on disease severity and age ( $<v s .>18$ years): either two units (or pediatric equivalent) of high titer $(\geq 1: 80)$ or low titer $(\leq 1: 10)$ anti-influenza virus hemagglutinin antibodies CP; 28 days of follow-up. Objectives: efficacy of high-titer $v$ s. low-titer CP.
(SD, 7) and had had ARDS for a mean of 6 days (SD 3) prior to receiving CP. Three patients (6.5\%) died within 7 days. Among survivors, $\mathrm{PaO}_{2} / \mathrm{FiO}_{2}$ increased by 112 units (95\% CI: 82-142); severity of radiological signs decreased in 23\% ( $95 \%$ CI: 5-42\%); CRP, ferritin and lactate dehydrogenase levels decreased by $60 \%, 36 \%$ and $20 \%$, respectively. Weaning from CPAP was achieved in 26/30 patients and $3 / 7$ intubated patients could be extubated. Five serious adverse events occurred in four patients (1 TRALI), of which two were possibly treatment related.

$92 / 138$ randomized to the high-titer, $48 / 138$ to the low-titer group. At baseline, 60 (43\%) participants were in intensive care; 55/78 (71\%) of participants were not in intensive care requiring oxygen. Early termination. No superiority of high-titer over low-titer plasma (OR on day 7: 1.22; 95\% CI: 0.65-2.29, $P=0.54$ ). $34 \%$ of participants (47/138) experienced 88 serious adverse events, including ARDS. Ten patients died (6 [7\%] in the high-titer group, 4 [9\%] in the low-titer group, $P=0.73$ ), worsening of ARDS was the most common cause of death. 


\section{Virus, Refl(s)}

\section{Influenza $A$ or $B \boldsymbol{B}$}

Lancet Respir Med.

2019;7(11):951-963.

Influenza $A$ or $B \xi$

Lancet Respir Med.

2017;5(6):500-511

\section{Influenza A (H1N1) ${ }^{\circ \wedge}$}

Clin Infect Dis 2011:52:447-456.

Influenza A (H1N1) ${ }^{\circ}$

Chest. 2013:144(2):464-473.

\section{Ebola virus disease ${ }^{\wedge} \wedge$}

N Engl J Med. 2016;374(1):33-42,

N Engl J Med. 2017;376(13):1297.

Ebola virus disease ${ }^{\wedge \wedge}$ \&

J Infect. 2017;74(3):302-309.

Case series. Patients treated $v$ s those who did not receive 1 unit

SARS-CoV-1 $* \S \S$

Clin Microbiol Infect.

2004;10(7):676-678.

SARS-CoV- $1 * \$ \$$

Eur J Clin Microbiol Infect Dis. 2005:24(1):44-46. (450 mL) of ABO-compatible convalescent whole blood (CWB) within the first $24 \mathrm{~h}$ of admission over a period of 1-4 $\mathrm{h}$.

Retrospective. 19 patients (38.7 \pm 12.39 years old) given $200-400 \mathrm{~mL}$ CP (coronavirus titer range 1:160-1: 2560) compared to 21 patients (47.9 919.60 years old) given methylprednisolone pulses.

Retrospective. Patients ( $\mathrm{n}=33 / 80$ ) given $\mathrm{CP}$ (median volume

\section{Randomized, double-blind, placebo-controlled trial. Adults} ( $\geq 18$ years of age), confirmed severe influenza A or B infection needing hospital treatment, symptoms starting within 7 days before randomization, assigned to standard care (which included antiviral therapy) + either $500 \mathrm{~mL}$ infusion of high-titer H-Is ( $0.25 \mathrm{~g} / \mathrm{kg}$ bodyweight, $24.75 \mathrm{~g}$ maximum) or saline placebo. Primary outcome: clinical status at day 7.

Randomized, open-label, multicenter, phase II retrospective trial. Hospitalized patients with severe (hypoxia/tachypnea) influenza A or B assigned on day 0 to standard care \pm 2 units (225-350 mL/unit or $8 \mathrm{~mL} / \mathrm{kg}$ pediatric equivalent) of compatible anti-influenza plasma hemagglutination inhibition antibody titers $\geq 1: 80$ CP, follow-up: 28 days. Objectives: time to normalization of respiratory status by day 28 of hospitalization.

Prospective cohort study. Within 7 days of symptom onset, 93 patients $\geq 18$ years old with severe H1N1 2009 infection needing intensive care were given the possibility of a $500 \mathrm{~mL}$ $\mathrm{CP}$ infusion over a $4 \mathrm{~h}$ period (neutralizing antibody titer $\geq 1: 160$ ).

Multicenter, prospective, double-blind, randomized controlled trial. Patients with severe H1N1 infection on standard antiviral treatment requiring intensive care and ventilatory support randomized to receive one dose of $0.4 \mathrm{~g} / \mathrm{kg}$ of $\mathrm{H}$-Ig (17 patients) or $0.4 \mathrm{~g} / \mathrm{kg}$ normal intravenous immunoglobulins (18 matched patients) over a period of $4 \mathrm{~h}$.

Non-randomized, comparative study. Patients of various ages, confirmed EVD, two consecutive transfusions of 200 to $250 \mathrm{~mL}$ of $\mathrm{ABO}$-compatible $\mathrm{CP}$ with varying levels of neutralizing antibodies. Transfusions initiated up to 2 days after diagnosis. Controls: 418 patients who had been treated at the same center during the previous 5 months. Primary outcome: risk of death from 3 to 16 days after diagnosis, adjustments for age; patients who died before day 3 excluded.

$279 \mathrm{~mL}$, coronavirus titer range, 1:160-1:2,560) within day 14 after the onset of symptoms $v$ s. patients given CP more than 14 days after hospital admission.
Results/Interpretation/Limitations

156 received $500 \mathrm{~mL}$ of $\mathrm{H}-\mathrm{Ig}$, 152, placebo (224/308 influenza A serotypes and 84/308 influenza B serotypes). Clear rise in hemagglutination inhibition titers against influenza A; smaller rise in influenza $B$ titers in the treated group. In subgroup analyses, the OR was 0.94 (0.55-1.59) in patients with influenza A and 3.19 (1.21-8.42) in those with influenza B (interaction $P=0.023)$. Through 28 days of follow-up, 47/156 (30\%) of patients in the H-Ig group and 45/152 (30\%) in the placebo group died or experienced a serious adverse event (HR 1.06, 95\% CI: 0.70-1.60; $P=0.79$ ).

Respiratory status normalized in 28/42 (67\%) of the participants in the plasma group $v$ s. 24/45 (53\%) in the standard care alone group (HR: $1.71,95 \% \mathrm{CI}: 0.96-3.06, P=0.069)$. One patient in the plasma group, and five (10\%) in the standard care group died (HR 0.19 [95\% CI: 0.02-1.65], $P=0.093$ ). No difference between groups in days of hospital stay (median, 6 days $v s$. 11 days, $P=0.13$ ) or in mechanical ventilation (median, 0 days $v s .3$ days, $P=0.14$ ). Serious adverse events including ARDS lower in the plasma group than in the standard care group ( $9 / 46$ [20\%] vs. $20 / 52[38 \%], P=0.041$.

20/93 patients (21.5\%) received CP. Mortality was lower in the treatment group than in the matched control group (20.0\% vs $54.8 \% ; P=0.01$, OR, $0.20 ; 95 \%$ CI: $.06-.69 ; P=0.011$ ). CP-treated individuals showed significantly lower viral loads, and post-treatment levels of interleukin-6, interleukin-10 and tumor necrosis factor- $\alpha$ than those who refused $\mathrm{CP}$ treatment $(P<0.05)$.

Patients receiving $\mathrm{H}-\mathrm{Ig}$ had lower post-treatment (days 5 and 7 ) viral loads than controls ( $P=0.04$ and $P=0.02$, respectively). Initial serum cytokine level, significantly higher in the H-Ig group, fell to control levels 3 days after treatment. In patients receiving treatment within 5 days of symptom onset, H-Ig was the only factor related to mortality ( $0 \%$ us. $40 \%$, respectively, OR, $0.14 ; 95 \% \mathrm{CI}: 0.02-0.92 ; P=0.04$ ). No treatment-related adverse events.

84 patients treated with plasma included in the analysis. From day 3 to day 16 after diagnosis, the risk of death was $31 \%$ in the CP group vs. 38\% in the control group (risk difference, -7\%; 95\% CI: -18 to 4). No serious adverse reactions were associated with the use of CP. CP treatment was not associated with a significant improvement in survival, in patients with confirmed EVD. However: (i) three-fourths of CP donors had low titers of neutralizing antibodies (1:10-1:40), only a minority (5\%) had higher titers (1:160), and (ii) patients receiving plasma with high doses anti-Ebola virus IgG antibodies had larger decreases in viral loads.

Compared with 25 non-treated patients, improved survival was documented in 44 subjects who received CWB (deaths, $44 \%$ vs. $27.9 \%$, respectively; odds ratio: $2.3,95 \%$ CI: $0.8-6.5$ ). There were significant difference between viral load on admission and after $24 \mathrm{~h}$ of treatment with CWB $(P<0.01)$. No adverse events.

Discharge at the end of a 3 -week hospitalization in $74 \%$ of subjects receiving $\mathrm{CP}$ and in $19 \%$ of those on methylprednisolone. Mortality: 0/19 (CP group) vs. 5/21 (steroid group). Unknown titer or type of antibodies affecting outcomes. Anti-SARS-CoV-1 antibodies contained within the CP not standardized.

At completion of a 3-week hospitalization, compared to the overall SARS-related mortality for admitted patients $(17 \%, n=299)$, those receiving plasma earlier had a lower mortality rate (12.5\%). No adverse events reported. No correlation between clinical response and antibody titers or transfused volumes. 
\#Donor selection is critical for the safety of plasma and plasma products. For SARS-CoV-2 studies; the FDA established that plasma should be collected from patients who had recovered from COVID-19 who had a negative SARS-CoV-2 polymerase chain reaction test and had been symptom-free for at least 14 days. Donors should meet the eligibility criteria for standard blood donors as set out in federal regulation 21 CFR 630.10 and 21 CFR 630.15, and standard testing should follow regulation 21 CFR $610.40 . \S$ A meta-analysis of studies published between 1918 and 1925 on the so-called Spanish influenza pneumonia (Ann Intern Med.2006;145(8):599-609), revealed lower case-fatality rates in patients receiving CP than in controls (16\% vs.37\%,respectively); maximal benefit being found in those receiving early treatment compared to patients who were transfused later ( $19 \%$ vs. $59 \%$, respectively). $§ \S$ Case reports and case series concerning the Middle Eas respiratory syndrome coronavirus (MERS-CoV) responsible for the 2015 MERS did not show clinical improvement with the administration of CP, and raised the issue of the quality of plasma and the role of neutralizing antibody titers in studies of CP (Sci Rep. 2016;6:25359, Emerg Infect Dis. 2015;21(12):2186-2189). ${ }^{\circ}$ Data from this prospective, non-randomized trial are in keeping with information of a contemporary retrospective observational study in the setting of H1N1 (Hong Kong Med J.2010;16(6):447-454). Comparable support stems from case reports and case series of the 2006 avian influenza A/H5N1 outbreak and the 2015 outbreak influenza A (H7N9) (Hong Kong Med J. 2006;12:489; N Engl J Med. 2007;357:1450-1451; Int J Infect Dis. 2015;41:3-5; PLoS One. $2008 ; 3:$ e2985). The latter studies also call for selecting convalescent donors based on the viral sequence of individual patients, a key issue for infections, such as influenza, caused by multiple strains of viruses. ${ }^{\wedge}$ The overall evidence that the administration of CP, serum, or H-Ig might be beneficial for the treatment of severe acute respiratory infections due to influenza or SARS-CoV-1 has been systematically reviewed and meta-analyzed (J Infect Dis.2015-211(1):80-90). As a whole, a 75\% reduction in the odds of mortality was found among patients with severe acute respiratory infections of viral etiology who were treated with convalescent plasma (or serum), together with a clinically relevant impact of the treatment on reducing the viral load. No evidence of serious adverse events or complications due to therapy were found and there was some evidence of a reduced use of critical care resources and length of hospital stay. Maximal reduction in mortality was achieved when CP was administered early after symptom onset. ^^The efficacy of CP in Ebola virus disease has been reported in two case series (Clin Infect Dis. 2015;61(4):496-502, N Engl J Med. 2016;374(7):636-646). \& In addition to Ebola virus disease, CP has been evaluated in the treatment of other viral hemorrhagic fevers e.g. Bolivian (Am J Med. 1966;40(2):217-230), Argentine (Presse Med. 1986;15(45):2239-2242.), and Lassa fever (Trans R Soc Trop Med Hyg. 1984;78(3):319-324). Inherent limitations and confounders compel caution in the analysis of these small studies. $\quad$ *Data from case reports (Transfus Apher Sci. 2003;29(1):101; Hong Kong Med J. 2003;9(3):199-201; Zhonghua Yi Xue Za Zhi. 2003;83(12):1018-1022) and case series (J Antimicrob Chemother.2005;56(5):919-922) are in keeping with information from retrospective, non-randomized studies, in this setting. SARS-CoV-2: severe acute respiratory syndrome of the coronavirus 2019, which causes COVID-19; SARS-CoV-1: 2003 severe acute respiratory syndrome-associated coronavirus 1 outbreak in Hong Kong; EVD: Ebola virus disease; ARDS: acute respiratory distress syndrome; $\mathrm{PaO}_{2} / \mathrm{FiO}_{2}$ : ratio of partial arterial oxygen pressure to fraction of inspired oxygten;TRALI: transfusion-related acute lung injury (whose features are similar to those of SARS-CoV2 infection); SOFA: Sequential Organ Failure Assessment; ECMO: extracorporeal membrane oxygenation support; CPAP: continuous positive airway pressure; H-Ig: hyperimmune immunoglobulin CP: convalescent plasma; CRP: C-reactive protein; OR: odds ratio; HR: hazard ratio; Cl: confidence interval; SD: standard deviation; PCR: polymerase chain reaction.

Table 2. Major emerging viruses potentially transmitted by blood and plasma products. ${ }^{\S}$

\begin{tabular}{|c|c|c|c|c|}
\hline Major pathogens & Description & Epidemiology & Detection method & Inactivation/elimination method \\
\hline \multicolumn{5}{|l|}{ Enveloped viruses } \\
\hline West Nile virus & Flavivirus causing encephalitis & South Europe, Africa, Americas & NAT, antibody IgM* & Solvent/detergent, heat \\
\hline Dengue virus & $\begin{array}{l}\text { Flavivirus causing myalgia, arthralgia, } \\
\text { hemorrhagic fever }\end{array}$ & South Asia, South America, Africa & NAT/antigen antibody. & Solvent/detergent, heat \\
\hline Chikungunya virus & Alphavirus causing arthralgia & South Asia, Africa & NAT/antibody. & Solvent/detergent, heat \\
\hline $\begin{array}{l}\text { Hepatitis B virus escape } \\
\text { variants }\end{array}$ & Causes hepatitis, hepatocarcinoma & Asia, Americas, Europe & NAT/sequence HBsAg. & Heat, vaccination. \\
\hline
\end{tabular}

\section{Non-enveloped viruses}

Parvoviruses

Enterovirus

Cause myalgia, anemia, fetal malformation Worldwide Picornaviruses causing pharyngitis, myalgia, Worldwide neuritis, encephalitis

Circoviruses No known disease

Unknown

$\begin{array}{ll}\text { NAT/antibody } & \text { Prolonged heat } \\ \text { NAT } & \text { Heat }\end{array}$

\section{Major emerging prions}

Variant Creutzfeldt-Jakob Prion infectivity in brain, lymphoid tissues, Europe, North America, Japan, disease prion and blood
Taiwan, Saudi Arabia.

\section{No screening test; diagnosis by histopathology and western blot of brain or tonsils after onset of symptoms}

$\S$ The term "Emerging" denotes those pathogens whose incidence in humans has increased or may increase in the future, based on their appearance in a new host population or changes in ep demiology in an existing population. This definition includes causative agent(s) leading to a new infection, a re-emerging infection, or an infection with new attributes (e.g., drug resistance or

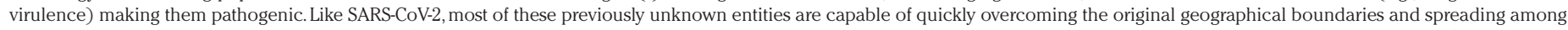

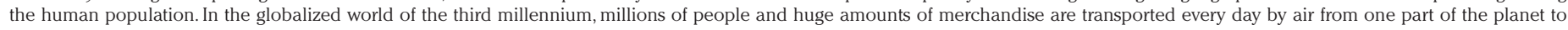
another.This facilitates the 'traffic' of microbes and the diseases they cause from ecological niches in which they were confined, and their rapid spread to every corner of the earth. Unpredictable global and national events (wars, world tourism, migration) can further increase the spread of blood-borne diseases. With regards to the roles of zoonotic transmission in the introduction of an

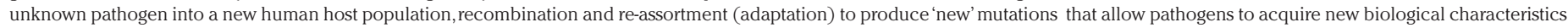

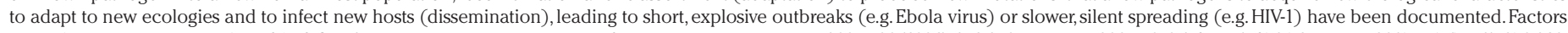

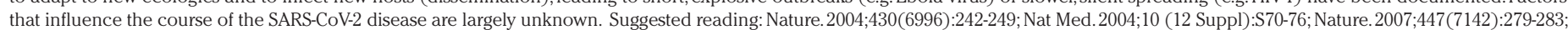
Nature. 2008;451(7181):990-993; Blood Transfus. 2009;7(3):167-171; Semin Thromb Hemost. 2013;39(7):779-793; Haematologica 2013;98(10):1495-1498. *Strategy currently only used in North America and Canada, ${ }^{\circ}$ Only four cases reported. IgM: immunoglobulin M; NAT: nucleic acid testing; PCR: polymerase chain reaction.

5,000 patients in another study. ${ }^{5}$ Also considering a risk/benefit analysis performed to improve the treatment of severe acute respiratory distress syndrome caused by SARS-CoV-2 infection, ${ }^{1}$ the Food and Drug Administration (FDA) issued guidance on CP collection and distribution in the USA and recommended conducting clinical trials with $\mathrm{CP}$ in the setting of SARS-CoV-2 infection. ${ }^{6}$ It has been proposed that, in such trials, one CP unit is used for post-expo- sure prophylaxis and one to two units for treatment of SARS-CoV-2 infection. ${ }^{1}$ For patients who fail to meet the criteria for enrollment in clinical trials, the FDA has approved protocols for emergency use and expanded access. ${ }^{4}$ In parallel, the plasma industry joined forces (the CoVIg-19 ALLIANCE) to increase plasma collection and produce safe and effective $\mathrm{CP}$ and hyperimmune immunoglobulins (H-Ig) ${ }^{7,8}$ Beside the USA, other countries 9 
are collecting $\mathrm{CP}$ to be used in SARS-CoV-2 infections, and many studies are ongoing. ${ }^{10}$

Parallel to the submission of the Italian study, an openlabel, multicenter, randomized trial from China appeared, in which patients with SARS-CoV-2 and severe acute respiratory distress syndrome were randomized to $4-13 \mathrm{~mL} / \mathrm{kg}$ of CP plus standard treatment vs. standard treatment alone (Table 1). The calculated sample size was 100 patients for each group. Due to the containment of the SARS-CoV-2 epidemic in China, the study was terminated when 103 of the 200 planned patients had been enrolled. At termination of the trial, improvement was found in 21/23 patients in the CP group vs. $15 / 22$ in the control group $(P=0.03)$ among those with severe disease, and in 6/29 of the CP group vs. $7 / 29$ in the control group $(P=0.83)$ among those with lifethreatening disease. There was no between-group difference in mortality $(P=0.30)$ and two adverse events were detected in two patients in the CP group.

While antibody administration by means of $\mathrm{CP}$ is indeed a reliable strategy for conferring immediate immunity against viral agents to individuals with SARS-CoV-2 infection, there is uncertainty about whether CP or $\mathrm{H}$-Ig is the more effective product to be administered..$^{10,11}$ While $\mathrm{CP}$ is characterized by donor-dependent variability in antibody specificity and titers, H-Ig contains standardized antibody concentrations. On the other hand, while the IgM fraction, a key weapon against some viruses, is removed from plasma during $\mathrm{H}-\mathrm{Ig}$ fractionation, $\mathrm{CP}$ also provides coagulation factors (to fight hemorrhagic fevers, such as Ebola). ${ }^{2}$ Although specific antibodies hamper viral replication, the SARS-CoV spike (S) protein is the main antigenic component responsible for biological effects, e.g., host immune responses, neutralizing-antibody formation, T-cell responses and ultimately protective immunity. ${ }^{12}$ On the whole, the proportion of anti-S protein antibodies, relationships between $\operatorname{IgG} / \operatorname{IgA} / \operatorname{IgM}$, standardization of antibody titers and optimal dosing and scheduling of $\mathrm{CP}$ administration are still major unknowns from studies conducted so far in the frame of the SARS-CoV-2 pandemic.

This scenario of growing interest from clinicians, patients, policy-makers, health systems and pharmaceutical industries provides an unprecedented opportunity to exert a major imprint on the practice of medicine. ${ }^{2}$ A concerted effort is warranted to achieve globally uniform, high-quality standards for $\mathrm{CP}$ or $\mathrm{H}$-Ig preparations. In high-income countries, the industrial production of plasma-derived products has never been safer than nowadays both because of the guidelines produced by the FDA and European Medicines Agency on donor selection and screening and because of the availability of viral inactivation methods. Plasma is collected at plasmapheresis centers using technologies regularly inspected by governing bodies before approval for commercial use. Plasma is screened after each donation and re-screened in mini-pools for human immunodeficiency virus-1, hepatitis A, B and C viruses, and parvovirus B19, and Plasma Master Files are subject to yearly approval by regulatory agencies. ${ }^{13}$ Once collected, plasma from single donors may be administered directly to patients or pooled to manufacture plasma-derived products such as $\mathrm{H}$-Ig, coagulation factors and others. The resulting products may be treated with solvent/detergent and/or super-heated (at $80^{\circ} \mathrm{C}$ for 3 days), pasteurized or nano-filtered. The aforementioned approaches are highly effective in minimizing pathogen transmission, as demonstrated by the fact that no blood-borne pathogen transmission has been reported since 1987 for commercially prepared plasma products received by patients with hemophilia, the epitome of multi-transfused patients. ${ }^{13}$ In theory, however, risks remain pertaining to emerging and re-emerging pathogens (prions, non-lipid enveloped viruses) (Table 2), for which diagnosis and inactivation methods are still a challenge. ${ }^{14}$ The reasons for this caveat concerning risks include the lack of reliable screening tests for some pathogens (e.g. prions), no screening for unknown pathogens, and relative poor sensitivity/specificity of the available assay methods. ${ }^{15}$ Furthermore, some viral mutants may escape screening, ${ }^{16}$ which may also not pick up potential plasma contamination from infectious but not yet seropositive donors. In addition, there may be low-level chronic carriers among donors who remain undetected and yet contribute to infect the plasma pool. ${ }^{17,18}$ Finally, determining the prevalence of emerging pathogens may be difficult when there is a long latency between infection and symptom onset. ${ }^{19}$

On this background and with these knowledge gaps, the adaptation of screening methods is a constant challenge, ${ }^{13}$ and public health organizations and plasma pharmaceutical industries have combined efforts to tackle the risks. In the framework of its global perspective, the World Health Organization tries to minimize pathogen transmission through early information and public health vigilance on the emergence of regional pathogens capable of causing transfusion-transmitted infections (e.g. Zika virus in Brazil), even before local authorities manage to develop means to prevent blood-borne transmission. ${ }^{20}$ Because 'zero risk' in terms of product safety is unlikely, governing bodies provide guidance to identify factors relevant for pathogen transmission. As an example, the presence of blood-borne hepatitis $\mathrm{E}$ virus may pose significant threats to some people (e.g., the elderly, immunocompromised individuals) despite being of low risk to other potential recipients. Thus, in addition to the circumstances under which blood products are collected and manufactured, the nature of the pathogen (e.g., its physical characteristics, level of virulence, prevalence) and the patients' characteristics (age, immune status, geographical location, lifestyle, treatment urgency) should be considered when choosing the individual treatment (and assessing an acceptable level of risk).

Alongside this scenario of basically satisfactory blood product safety in high-income countries, it should be considered that in most low/middle-income countries procedures for blood collection are seldom standardized, and donor selection, screening and viral inactivation often fail to meet the criteria validated and implemented by regulatory agencies in high-income countries. If insufficient antiSARS-CoV-2 CP is available from high-income countries to meet global needs, the use of plasma from low/middleincome countries may become necessary but may also raise some issues, because the type and prevalence of infectious agents likely differ in different populations. ${ }^{13}$

To sum up, if worldwide uniform advancements in blood-banking quality are encouraged in low/middleincome countries, there is now a global opportunity to perform clinical studies on the efficacy of $\mathrm{CP}$ or $\mathrm{H}$-Ig in viral infections and address uncertainties on the occurrence of 
serious adverse events related to the administration of these products. ${ }^{10}$ Removing regulatory barriers that limit the use of pathogen-reduction technology for CP collections would be a major help in this respect. ${ }^{2}$ The process of obtaining informed consent requires communication of risks and benefits of treatments to patients. SARS-CoV-2 is an easily inactivated enveloped virus, ${ }^{13}$ and strict regulations for plasma product manufacturing minimize the risk of known and unknown pathogens. Apart from emergency situations, the extent to which people should be further informed on specific risks associated with any particular product will depend on a variety of factors including availability of alternative treatments, and the patients' characteristics (e.g., age, physical/mental condition, education/level of understanding, language barriers/religious beliefs). A good understanding by health care professionals of the sources and modes of production of plasma derivatives and of pathogen-reduction/inactivation techniques might be an additional benefit of studies involving CP.

\section{Addendum}

Parallel to the submission of this Editorial, a systematic review of completed (as of June 4, 2020) and ongoing (n=98) studies on the efficacy and safety of convalescent plasma or hyperimmune immunoglobulins to reduce mortality in patients with SARSCoV-2 infection appeared (Cochrane Database Syst Rev. 2020;7(7):CD013600. Its provisional conclusions support the clinical relevance of the concepts summarized in the present report.

\section{References}

1. Bloch EM, Shoham S, Casadevall A, et al. Deployment of convalescent plasma for the prevention and treatment of COVID-19. J Clin Invest. 2020;130(6):2757-2765.

2. Roback JD, Guarner J. Convalescent plasma to treat COVID-19: possibilities and challenges. JAMA. 2020; 323(16):1561-1562.

3. Perotti C, Baldanti F, Bruno R et al. Mortality reduction in 46 severe Covid-19 patients treated with hyperimmune plasma. A proof of concept single arm multicenter trial. Haematologica. 2020;105(12): 2834-2840.

4. Semple JW, Rebetz J, Kapur R. Transfusion-associated circulatory over- load and transfusion-related acute lung injury. Blood. 2019;133 (17):1840-1853.

5. Joyner M, Wright RS, Fairweather D, et al. Early safety indicators of COVID-19 convalescent plasma in 5,000 patients. medRxiv. 2020 May 14. [Epub ahead of print]

6. Food and Drug Administration. Recommendations for Investigational COVID-19 Convalescent Plasma. Available from https://www.fda.gov/vaccines-blood-biologics/investigational-newdrug-ind-or-device-exemption-ide-process-cber/recommendationsinvestigational-covid-19-convalescent-plasma\#Collection $\% 20$ of\%20COVID-19 [Accessed May 9, 2020]

7. https:/clinicaltrials.gov/ct2/show/NCT04488081?term $=i+$ spy+covid \&draw $=2 \&$ rank $=1$ [Accessed August 11, 2020] 7 bis.

8. https://www.covidrdalliance.com/pdf/ISPY_PressRelease August_3_Final.pdf

9. Sanquin. Sanquin starts collecting plasma from cured corona patients. Available from: https://www.sanquin.org/news/2020/mar/sanquinstarts-collecting-plasma-from-cured-corona-patients. [Accessed May 9 , 2020]

10. Piechotta V, Chai KL, Valk SJ, et al. Convalescent plasma or hyperimmune immunoglobulin for people with COVID-19: a living systematic review. Cochrane Database Syst Rev. 2020;7:CD013600.

11. [Editorial: no authors listed]. The resurgence of convalescent plasma therapy. Lancet Haematol. 2020;7(5):e353.

12. Du L, He Y, Zhou Y, Liu S, Zheng BJ, Jiang S. The spike protein of SARS-CoV--a target for vaccine and therapeutic development. Nat Rev Microbiol. 2009;7(3):226-236.

13. Di Minno G, Navarro D, Perno CF, et al. Pathogen reduction/inactivation of products for the treatment of bleeding disorders: what are the processes and what should we say to patients? Ann Hematol. 2017;96(8):1253-1270

14. Srivastava A, Brewer AK, Mauser-Bunschoten EP, et al. Guidelines for the management of hemophilia. Haemophilia. 2013;19(1):e1-47.

15. Di Minno G, Canaro M, Ironside JW, et al. Pathogen safety of longterm treatments for bleeding disorders: still relevant to current practice. Haematologica. 2013;98(10):1495-1498.

16. Salpini R, Piermatteo L, Battisti A, et al. A hyper-glycosylation of HBV surface antigen correlates with HBsAg-negativity at immunosuppression-driven $\mathrm{HBV}$ reactivation in vivo and hinders $\mathrm{HBs} A g$ recognition in vitro. Viruses. 2020;12(2):251.

17. Schreiber GB, Busch MP, Kleinman SH, Korelitz JJ. The risk of transfusion-transmitted viral infections. The Retrovirus Epidemiology Donor Study. N Engl J Med. 1996;334(26):1685-1690.

18. Di Minno G, Perno CF, Tiede A, et al. Current concepts in the prevention of pathogen transmission via blood/plasma-derived products for bleeding disorders. Blood Rev. 2016;30(1):35-48.

19. Ludlam CA, Powderly WG, Bozzette S, et al. Clinical perspectives of emerging pathogens in bleeding disorders. Lancet. 2006;367(9506):252261.

20. Barjas-Castro ML, Angerami RN, Cunha MS, et al. Probable transfusion-transmitted Zika virus in Brazil. Transfusion. 2016;56(7):16841688. 